\title{
La co-docencia en el contexto universitario como estrategia para la innovación docente
}

\section{Castro Zubizarreta, Ana ${ }^{a}$; Briones Pérez, Elena ${ }^{b}$, Izquierdo Magaldi, Belén} ${ }^{a}$ Universidad de Cantabria (castroza@unican.es), ${ }^{\mathrm{b}}$ Universidad de Cantabria (elena.briones@unican.es) y ${ }^{\mathrm{c}}$ Universidad de Cantabria (mariabelen.izquierdo@unican.es)

\begin{abstract}
Co-teaching has been described as one of the successful educational actions in the university context. It implies practices of cooperative teaching developed by two or more teachers generating a constant feedback for the teachers involved, sharing their experience and knowledge during their classes.

This paper presents the design and evaluation of a co-teaching experience developed at the Faculty of Education of the University of Cantabria. This educational experience is designed as a modality of co-teaching as station teaching and mentoring among students. The initial expectations, the benefits derived from this educational practice, the difficulties encountered and the proposals for improvement for this teaching practice are approached from the perspective of the teachers involved in the development of the co-teaching designed. Some learning outcomes are also described in the students. The results of this project show the positive evaluation of the experience and the commitment to this type of activities in spite of some temporal and organizational limitations.
\end{abstract}

Keywords: Co-teaching, educational innovation, Higher Education, methodology, mentoring.

\section{Resumen}

La co-docencia ha sido descrita como una de las actuaciones educativas de éxito en el contexto universitario. Supone prácticas de enseñanza cooperativa desarrollada por dos o más docentes generando un marco de retroalimentación constante para el profesorado implicado, al compartir su experiencia y conocimientos durante el desarrollo de las clases.

En este trabajo se presenta el diseño y valoración de una experiencia de codocencia desarrollada en la Facultad de Educación de la Universidad de Cantabria. Esta experiencia educativa se diseña como una modalidad de codocencia en estaciones a través de la mentoría entre estudiantes. Se abordan desde la perspectiva de las docentes implicadas en el desarrollo de la codocencia, sus expectativas iniciales, los beneficios que según ellas se deriva de esta práctica educativa, las dificultaddes encontradas y las propuestas de mejora para la continuidad de esta práctica docente. Asímismo se recogen algunos resultados de aprendizaje en los estudiantes. Los resultados del 
trabajo ponen de manifiesto la valoración positiva de la experiencia y el compromiso de las docentes con este tipo de actividades a pesar de las limitaciones temporales y organizativas encontradas.

Palabras clave: co-docencia, innovación educativa, Educación Superior, metodología, mentoría.

\section{Introducción}

La co-docencia o co-enseñanza ha sido considerada durante años, siguiendo a Suárez-Díaz (2016), como una estrategia eficaz para fortalecer la inclusión de alumnado con necesidades específicas de apoyo educativo en las aulas escolares. En el ámbito de la Educación Superior encontramos algunas experiencias de co-docencia que, insertadas bajo prácticas de innovación docente, se desarrollan con éxito en esta etapa educativa (Picheira y Otondo, 2016; Suárez-Díaz, 2016). En esta línea de desarrollo de prácticas de innovación docente en la Educación Superior, presentamos en este trabajo, un diseño de codocencia y la valoración de su práctica desarrollada en la Facultad de Educación de la Universidad de Cantabria durante el curso 2016-17 y que se enmarca dentro de un proyecto de innovación docente que lleva por título: "Co-docencia y Mentoría en el contexto universitario. Una propuesta metodológica para la coordinación docente y el aprendizaje activo del alumnado" aprobado en la III Convocatoria de Proyectos de innovación docente de la Universidad de Cantabria.

La co-docencia ha sido descrita como una de las actuaciones educativas de éxito (AEE) en el contexto universitario (Flecha et al., 2014). De hecho, tal y como señalan Cook y Friend (1995) la co-docencia ya fue recomendada tanto en Inglaterra como en Estados Unidos en los años sesenta como estrategia para reorganizar la Educación Secundaria. A pesar de este antecedente tan temprano de su uso en la escuela, su desarrollo sigue siendo menor en las aulas universitarias.

El término co-docencia también conocido como co-enseñanza o co-teaching define aquellas prácticas de enseñanza cooperativa desarrollada por dos o más docentes (Beamish, Bryer y Davies, 2006). La co-docencia favorece la generación de un marco de retroalimentación constante para el profesorado implicado, al compartir su experiencia y conocimientos durante el desarrollo de las clases (Rodríguez, 2014). Al respecto, la coordinación, el consenso de objetivos, la alternancia de roles y la interdependencia son elementos clave en esta práctica educativa pues, tal y como señalan Chanmugam y Gerlach (2013), requiere de la participación igualitaria de los docentes durante la planificación de la docencia, la instrucción y el proceso de evaluación. Estos elementos, co-planificación, co-instrucción y co-evaluación según Conderman y Hedin (2012), se convierten en ejes vertebradores para la aplicación de cualquier práctica de co-docencia.

(cc) EY-NC-ND 2017, Universitat Politècnica de València 
Se han planteado además, unos principios que orientan las prácticas de co-docencia. Siguiendo las orientaciones de Villa et al. (2008) y Rodríguez (2014) concluimos las siguientes:

1. La coordinación del trabajo de los docentes para el logro de objetivos comunes.

2. El reconocimiento de la valía del otro, su experiencia y conocimiento.

3. La alternancia de roles entre los docentes participantes y la interdependencia para el logro de los objetivos comunes.

4. La utilización de un liderazgo compartido entre los co-docentes.

5. El carácter voluntario de la participación en la co-docencia y el compromiso individual de todos los docentes participantes.

Chanmugam y Gerlach (2013) destacan como beneficio potencial de la co-docencia para el propio profesorado participante, la posibilidad de conocer otras perspectivas, o formas de pensar, facilitando el intercambio de reflexiones y pareceres en un diálogo abierto donde el docente tiene la oportunidad de compartir impresiones que de otra forma hubieran quedado en una reflexión introspectiva.

Compartir la docencia según Crow y Smith (2005) y Rodríguez (2014), amplía la formación del profesorado participante y mejora su competencia interpersonal. En este sentido, Tobin y Roth, (2005) afirman que co-docencia es aprender en la práctica, dado que durante ella los docentes comparten sus competencias, habilidades, estilos docentes y estrategias metodológicas para el desarrollo de la docencia a un mismo grupo de estudiantes. A nuestro entender, la co-docencia es garante de coordinación, dada la necesidad de colaboración constante favoreciendo la integración interdisciplinar de los docentes.

Otros beneficios a destacar de la co-docencia en el ámbito universitario son los apuntados por Bekerman y Dankner (2010) quienes refieren una visión positiva de los alumnos respecto a la solvencia y coherencia de los co-educadores. Concretamente, estos autores demuestran que la retroalimentación aportada por el profesorado implicado creaba desde la perspectiva del alumnado mayores oportunidades de aprendizaje y enriquecimiento personal.

En todo caso, la co-docencia, también presenta aspectos limitadores, o dificultades en su práctica tal y como apuntan Castro y Figueroa (2006); Ginther, Phillips, y Grinseki (2007) y Rodríguez (2014). Entre las dificultades identifacadas por estos autores destacan el factor tiempo y la ausencia de espacios para planificar e implementar los programas; la ausencia de coordinación, el trabajo pedagógico aislado, la resistencia al trabajo colaborativo o la presencia de sentimientos de desconfianza o inseguridad.

A pesar de los aspectos limitadores anteriormente apuntados, las bonanzas que se desprenden del uso de la co-docencia en el ámbito universitario han alentado el desarrollo del proyecto y animado al profesorado participante a su desarrollo en las aulas. 


\subsection{Diferentes enfoques de co-docencia pero un mismo propósito: la enseñanza colaborativa}

La co-docencia al igual que tiene otros términos para referirse a ella como co-enseñanza, co-teaching, enseñanza colaborativa, enseñanza en equipo, cátedra compartida (SuárezDíaz, 2016) tiene también diferentes enfoques o tipos a la hora de llevarla a la práctica.

A partir de la revisión realizada por Rodríguez (2014) de los modelos de co-docencia propuestos por Friend y Cook (1996), y Hughes y Murawski (2001) a continuación presentamos una síntesis de las aportaciones de estos autores (Tabla 1), que se concreta en una tipología basada en el grado de interacción entre los docentes participantes y los estudiantes en escala ascendente:

Tabla 1. Modelos de co-docencia

\begin{tabular}{|l|l|}
\multicolumn{2}{|c|}{ MODELOS DE CO-DOCENCIA } \\
\hline \multicolumn{1}{|c|}{ TIPOLOGÍA } & \multicolumn{1}{c|}{ DESCRIPCIÓN } \\
\hline $\begin{array}{l}\text { Uno enseña, el otro observa o } \\
\text { Co-docencia de observación. }\end{array}$ & $\begin{array}{l}\text { Mientras uno enseña el otro recoge información de interés. } \\
\text { Requiere la coordinación en la información a recoger y el análisis } \\
\text { posterior de la información. }\end{array}$ \\
\hline $\begin{array}{l}\text { Uno enseña, el otro circula o } \\
\text { Co-docencia de apoyo. }\end{array}$ & $\begin{array}{l}\text { Mientras uno es el responsable principal de la enseñanza el otro } \\
\text { profesor circula ofreciendo ayuda puntual al estudiante que lo } \\
\text { demande. }\end{array}$ \\
\hline $\begin{array}{l}\text { Co-docencia en grupos } \\
\text { simultáneos o Enseñanza } \\
\text { paralela. }\end{array}$ & $\begin{array}{l}\text { Los profesores enseñan y comparten los mismos materiales } \\
\text { didácticos, pero dividen la clase en dos y asisten simultáneamente a } \\
\text { los estudiantes. }\end{array}$ \\
\hline $\begin{array}{l}\text { Co-docencia en rotación } \\
\text { con/sin Estaciones de } \\
\text { enseñanza }\end{array}$ & $\begin{array}{l}\text { Los profesores dividen el contenido y la clase en dos, cada uno } \\
\text { enseña el contenido que le correspondió a su grupo, luego el mismo } \\
\text { contenido al otro. Además, se puede establecer un tercer grupo } \\
\text { "estación" para que los estudiantes trabajen independientemente. }\end{array}$ \\
\hline $\begin{array}{l}\text { Co-docencia complementaria } \\
\text { Enseñanza alternativa o } \\
\text { diferenciada }\end{array}$ & $\begin{array}{l}\text { Consiste en que un profesor del equipo realiza acciones } \\
\text { para mejorar o complementar la enseñanza provista por el otro } \\
\text { profesor (e.g. parafraseo, enseñanza con un estilo diferente, enseñar } \\
\text { previamente las habilidades sociales requeridas para el aprendizaje } \\
\text { cooperativo). }\end{array}$ \\
\hline Equipo docente & $\begin{array}{l}\text { Un profesor es responsable del grupo mas amplio, y el otro del más } \\
\text { pequeño. }\end{array}$ \\
\hline $\begin{array}{l}\text { Los docentes brindan la misma instrucción de forma simultánea, en } \\
\text { un flujo de acción invisible que impide distinguir a un líder. Es el } \\
\text { enfoque más complejo y que más se ajusta a la idea de co- } \\
\text { enseñanza. }\end{array}$ \\
\hline
\end{tabular}

Esta diversidad de enfoques o modalidades de co-docencia responde a los distintos objetivos en el proceso de enseñanza-aprendizaje. Así, lo realmente interesante es ver cómo la co-docencia se erige como una propuesta metodológica flexible y abierta a las necesidades docentes y alumnado, pudiéndose generar nuevas fórmulas o variantes. 


\section{Objetivos}

Los objetivos que orientan el proyecto son los siguientes:

- Proponer y desarrollar una docencia innovadora en la Universidad de Cantabria utilizando actuaciones educativas de éxito (AEE) en el contexto universitario, optando en nuestro proyecto por la co-docencia y la mentoría entre el alumnado.

- Facilitar al alumnado la articulación de conocimientos teóricos y prácticos impartidos desde ramas epistemológicas diferentes ampliando la visión y perspectiva sobre un mismo foco temático y favoreciendo así un aprendizaje globalizado.

- Generar prácticas de trabajo cooperativo entre el alumnado a través de la mentoría entre alumnos de tres cursos del Grado en Educación Infantil que facilite un conocimiento más profundo del plan de estudios, organización y conexiones, así como potenciar las relaciones sociales entre el alumnado.

- Generar procesos de mejora de la coordinación docente y articulación de asignaturas a través de la co-docencia y el aprendizaje dialógico.

\section{Desarrollo de la innovación}

\subsection{Marco contextual de la experiencia: tres docentes, tres asignaturas distintas y un mismo plan de estudios}

El desarrollo de la co-docencia en equipo supone prestar en primer lugar atención a las personas implicadas en dicha práctica. Este aspecto requiere como hemos comentado anteriormente voluntariedad, pero sobre todo, conocimiento mutuo, diálogo, intercambio y la retroalimentación constante (Álvarez-Arregui, Rodríguez, Madrigal, Grossi y Arreguit, 2017). En el proyecto desarrollado, atendiendo a estas premisas, se coordinaron tres asignaturas, pertenecientes a tres áreas epistemológicas distintas impartidas por profesoras diferentes. Una asignatura de $1^{\circ}$ curso del Grado de Educación Infantil, Fundamentos teóricos de la Educación Infantil, con 43 estudiantes, impartida por el área de Teoría e historia de la educación. Una asignatura de $2^{\circ}$ curso, Psicopedagogía de la expresión y de la comunicación con 45 estudiantes, impartida por el área de Psicología del desarrollo y de la educación, y una asignatura de $3^{\circ}$ curso, Contextos didácticos y organizativos de la educación infantil, con 44 estudiantes, impartida desde el área de Didáctica y organización escolar. Las tres asignaturas presentan nexos de unión, en los contenidos de sus programas que conllevan una gradualidad en complejidad y desarrollo, de ahí, su ubicación temporal en el plan de estudios.

Concretamente, en la asignatura Fundamentos teóricos de la Educación Infantil ( $1^{\circ}$ curso) se abordan entre otros contenidos, la transición educativa, en la que desde una perspectiva teórica, sustentada en el modelo de la teoría crítica se identifican las discontinuidades entre las etapas educativas de Educación Infantil y Educación Primaria, así como, el derecho de participación de los niños en el proceso de transición. Asimismo, el fenómeno de la transición vuelve a ser recogido en la asignatura Psicopedagogía de la expresión y de la comunicación en el $2^{\circ}$ curso del Grado de Educación Infantil ligado a los procesos de alfabetización inicial y escolar que se producen en la Educación Infantil y se continúan y afianzan en la Educación Primaria. Finalmente, en $3^{\circ}$ se abordan en la asignatura Contextos didácticos y organizativos de la educación infantil, las nuevas alfabetizaciones, que entendemos como la transición que el niño realiza de la alfabetización inicial y escolar a la 
alfabetización en medios y digital.

Las docentes de las tres asignaturas realizaron prácticas de co-docencia en el grupo de $1^{\circ}$ donde iban introduciéndose desde el área de conocimiento de las profesoras implicadas, información relevante sobre el contenido objeto de estudio (la transición educativa). Se plantea por tanto, una práctica de co-docencia con base en el grupo de $1^{\text {er }}$ curso con estaciones de mentoría junto con los estudiantes de $2^{\circ}$ y $3^{\circ}$ curso del Grado de Magisterio de Educación Infantil, que favorezca tanto la coordinación docente como la percepción por parte del alumnado de los vínculos existentes entre las asignaturas, su gradualidad y posibilidades de relación de su contenido con la realidad educativa actual.

\subsection{Diseño de la experiencia}

Para el desarrollo de la experiencia planteamos un diseño metodológico que denominamos co-docencia en estaciones de mentoría que se desarrolla en dos fases, descrito en la Tabla 2. En cada una de estas fases se combina el enfoque de co-docencia de equipo docente, con la co-docencia en estaciones y la mentoría entre estudiantes. En las dos fases se trabaja con los estudiantes de primero, que reciben la co-docencia, es decir, las docentes brindan la misma instrucción de forma simultánea al mismo grupo de alumnos (véanse los contenidos en la Tabla 2). Además, se contempla la estación, que consiste en la mentoría entre estudiantes de primero y de segundo en la primera fase, y de estudiantes de primero y de tercero en la segunda fase. Esta agrupación entre estudiantes responde a la temática trabajada.

La singularidad de este diseño radica en que el proceso de enseñanza se plantea a través de la mentoría entre estudiantes por favorecer "un proceso de feed-back continuo de ayuda y orientación entre el mentor (alumno de curso superior que atesora los conocimientos y habilidades necesarias para ayudar), y un estudiante o un grupo de estudiantes de nuevo ingreso con la finalidad de paliar las necesidades de estos y optimizar su desarrollo y potencial de aprendizaje" (Valverde, Ruiz de Miguel, García Jímenez y Romero, 2004; p. 92).

De este modo, el diseño de la experiencia comprendió el desarrollo de co-docencia en modalidad de equipo docente y estaciones de mentorías desarrolladas por los estudiantes de cursos superiores al alumnado de primer curso.

Tabla 2. Diseño metodológico de la experiencia de co-docencia en el contexto universitario

\begin{tabular}{|c|c|c|}
\hline \multicolumn{3}{|c|}{ DISEÑO METODOLÓGICO } \\
\hline $\begin{array}{c}\text { CO-DOCENCIA EN } \\
\text { EQUIPO }\end{array}$ & 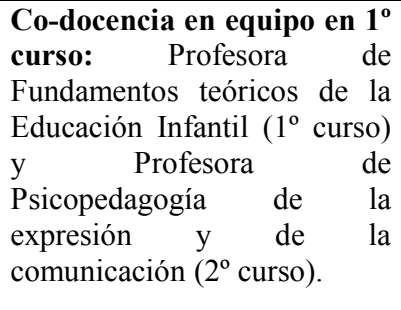 & $\begin{array}{l}\text { Contenido trabajado: } \\
\text { transición de educación infantil a la } \\
\text { educación primaria y la } \\
\text { alfabetización básica (habilidades de } \\
\text { lecto-escritura). } \\
\text { Técnicas para abordar el } \\
\text { contenido: ideas previas, clase } \\
\text { magistral participativa, interrogantes }\end{array}$ \\
\hline
\end{tabular}

(cc) EY-NC-ND 2017, Universitat Politècnica de València 


\begin{tabular}{|c|c|c|}
\hline $\begin{array}{c}\text { Y } \\
\text { ESTACIÓN DE } \\
\text { MENTORÍA } \\
\left(\mathbf{1}^{\mathbf{0}} \text { y } \mathbf{2}^{\mathbf{o}} \text { curso del Grado }\right. \\
\text { de Magisterio en } \\
\text { Educación Infantil }) \\
\text { Temporalización: } 2 \text { horas }\end{array}$ & $\begin{array}{l}\text { Mentoría del alumnado de } 2^{\circ} \\
\text { curso que de la asignatura } \\
\text { Psicopedagogía de la } \\
\text { expresión y de la } \\
\text { comunicación al alumnado de } \\
1^{\circ} \text { curso de la asignatura de } \\
\text { Fundamentos Teóricos de la } \\
\text { Educación Infantil. }\end{array}$ & $\begin{array}{l}\text { Contenido trabajado: la transición } \\
\text { educativa de Educación Infantil a } \\
\text { Educación Primaria y el proceso de } \\
\text { adquisición de las habilidades lecto- } \\
\text { escritoras ante la escolaridad } \\
\text { obligatoria. } \\
\text { Técnicas/actividades: Recuerdos } \\
\text { de la transición escolar y } \\
\text { experiencias vivenciadas por el } \\
\text { alumnado de } 2^{\circ} \text { sobre la transición } \\
\text { educativa y la adquisición de los } \\
\text { niños de las habilidades de lecto- } \\
\text { escritura percibida a raíz de sus } \\
\text { prácticas de enseñanza. }\end{array}$ \\
\hline $2^{\circ}$ FASE & $\begin{array}{l}\text { Co-docencia en equipo en } \mathbf{1}^{\circ} \\
\text { curso: Profesora de } \\
\text { Fundamentos teóricos de la } \\
\text { Educación Infantil }\left(1^{\circ} \text { curso }\right) \\
\text { y Profesora de Contextos } \\
\text { didácticos y organizativos de } \\
\text { la educación infantil }\left(3^{\circ}\right. \\
\text { curso). }\end{array}$ & 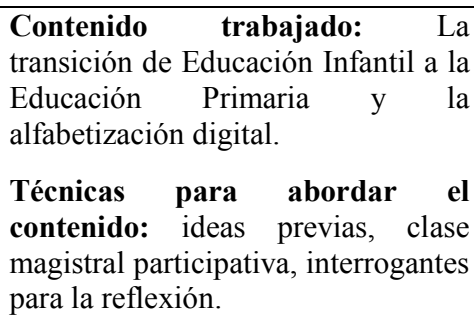 \\
\hline $\begin{array}{c}\text { EQUIPO } \\
\text { Y } \\
\text { ESTACIÓN DE } \\
\text { MENTORÍA } \\
\left(1^{\circ} \text { y } 3^{\circ} \text { curso del Grado }\right. \\
\text { de Magisterio en } \\
\text { Educación Infantil }) \\
\text { Temporalización: } 2 \text { horas }\end{array}$ & $\begin{array}{l}\text { Mentoría del alumnado de } 3^{\circ} \\
\text { curso que de la asignatura } \\
\text { Contextos didácticos y } \\
\text { organizativos de la educación } \\
\text { infantil, al alumnado de } 1^{\circ} \\
\text { curso de la asignatura de } \\
\text { Fundamentos Teóricos de la } \\
\text { Educación Infantil. }\end{array}$ & $\begin{array}{l}\text { Contenido trabajado: } \\
\text { transición de Educación Infantil a la } \\
\text { Educación Primaria y la } \\
\text { alfabetización digital. } \\
\text { Técnicas/actividades: Trabajo por } \\
\text { proyectos. Explicación de los } \\
\text { alumnos de } 3^{\circ} \text { a los de } 1^{\circ} \text { que } \\
\text { entienden por competencia } \\
\text { mediática y digital, su promoción } \\
\text { desde la Educación Infantil. } \\
\text { Creación de un proyecto práctico } \\
\text { que integre dichas competencias en } \\
\text { las aulas de Educación Infantil. Los } \\
\text { estudiantes de } 1^{\circ} \text { aportaron } \\
\text { sugerencias, a plantearon } \\
\text { interrogantes y compartieron } \\
\text { pareceres durante este proceso de } \\
\text { mentoría. }\end{array}$ \\
\hline
\end{tabular}




\section{Resultados}

A continuación se presentan los resultados derivados del análisis de contenido realizado sobre la valoración de las tres profesoras responsables de la actividad de co-docencia explicada anteriormente. Las nueve preguntas realizadas a las profesoras una vez concluida la actividad de co-docencia nos permite identificar las expectativas, las dificultades y beneficios percibidos en la experiencia de innovación en co-docencia llevada a cabo, además del grado en el cual se valora su puesta en práctica en la Educación Superior. Asimismo, estas profesoras comparten su intención de compromiso con este tipo de actividad y sus propuestas para favorecer su desarrollo en el contexto universitario. Además, las cuestiones sobre aprendizajes adquiridos dirigidas a los estudiantes mentorizados y mentores (Castro, Izquierdo-Magaldi y García-Ruiz, 2017), nos permiten confirmar la percepción de las docentes.

\subsection{Expectativas iniciales y valoración final}

Del análisis de las expectativas iniciales de las profesoras identificamos cinco categorías con matiz positivo y tan sólo una con un carácter más negativo. Las primeras se refieren a la posibilidad de poner en práctica la co-docencia con otras compañeras, el conocimiento de la conexión entre las asignaturas consideradas, el reto personal que supone el trabajo conjunto con otros compañeros, el ofrecer a los estudiantes una transmisión del conocimiento en espiral y el poder comprobar los beneficios que suponen estas experiencias innovadoras en el proceso de enseñanza-aprendizaje. Así lo observamos en los siguientes comentarios:

"El inicio del proyecto supuso para mí un reto personal, el escuchar a las compañeras, conocer su manera de entender la docencia, su metodología, y su enfoque sobre el tema a tratar: la transición educativa"

"Esperaba comprobar cómo estas propuestas innovadoras mejoraban el proceso de enseñanza aprendizaje”

Pero no todas las expectativas iniciales fueron positivas, también se expresaron ciertas incertidumbres tales como la dificultad percibida de su puesta en marcha, en relación a su gestión de tiempos, a la dificultad de los contenidos, y la apertura de los estudiantes a estas propuestas, tal como se expresa en el siguiente comentario:

"Pensaba que hablarles de una asignatura e un curso superior, con contenidos relacionados con procesos de lectura y escritura iba a resultar lejano para el alumnado de primer curso".

En cuanto al balance final realizado por las profesoras sobre el grado en el que se cumplieron sus expectativas, nos encontramos que este se inclina hacia lo positivo junto con algún elemento que ha generado cierta inquietud. Entre los resultados positivos nos encontramos la posibilidad que ha ofrecido esta actividad de conocer los diferentes enfoques y perspectivas de las compañeras, la oportunidad de sentirse seguro y cómodo en el desarrollo de las actividades y la oportunidad que supone para la formación. También ha incidido en este balance positivo la actitud receptiva mostrada por los estudiantes. Los

(cc) EY-NC-ND 2017, Universitat Politècnica de València 
elementos que han generado cierto ruido en este balance positivo han sido la dedicación temporal destinada a la co-docencia, pues se ha considerado escaso. Por tanto, podríamos resumir el balance entre las expectativas iniciales y finales con la siguiente observación realizada por una de las profesoras:

"Afortunadamente, el resultado ha superado de forma positiva todas las expectativas que tenía. A pesar de la carga lectiva y de trabajo de los participantes, su actitud receptiva ante la experiencia, su participación y muestras de interés por los temas tratados han motivado la continuidad del proyecto".

\subsection{Dificultades de la experiencia de innovación en co-docencia y de su aplicación en la Educación Superior}

Cuatro han sido las categorías que se han extraído de la valoración de las profesoras sobre las dificultades encontradas en la experiencia de co-docencia desarrollada:

- La organización de tiempos y espacios de encuentros entre las co-docentes y también entre los estudiantes.

- Las actitudes hacia la apertura de la docencia a otros compañeros de otras asignaturas, pues se perciben dificultades para la escuchar y la percepción del enriquecimiento mutuo de la colaboración.

- La limitación en el número de actividades ofrecidas a los estudiantes, debido a la falta de tiempo.

- "El diseño de una evaluación que revierta en la mejora de la participación e implicación de los participantes, tanto docentes como estudiantes", en palabras de una de la profesoras.

En cuanto a las dificultades de la aplicación de la co-docencia en la Educación Superior, observamos semejantes categorías a las anteriormente mencionadas. Pues se vuelve a mencionar la dificultad de gestión de tiempos y espacios, unido a la rigidez de la temporalidad y fragmentación de los planes de estudio y a la necesidad de un buen diseño con objetivos claros en evaluación y resultados de aprendizaje. También se mencionan elementos actitudinales propios del docente como: "la cultura individualista a la hora de impartir docencia" y "compartir su trabajo y experiencia profesional", y por otra parte, la necesidad de compromiso para mantener el "esfuerzo y dedicación que conlleva una experiencia continuada" de este tipo. Esta requisito actitudinal se describe en la siguiente cita :

"En general, una experiencia de este tipo necesita de la compenetración de los docentes implicados, de tal manera que formen un tándem educativo en la interacción con sus estudiantes".

\subsection{Beneficios de la co-docencia para docentes y estudiantes}

Los beneficios expresados por y para las profesoras que han participado en la experiencia de co-docencia hacen referencia tanto a aspectos personales, como profesionales. Entre los primeros expresan ganancias en su seguridad y confianza, y desarrollo de capacidades tales 
como la implicación, la observación, la reflexión y la innovación. Entre las profesionales destacan la oportunidad de intercambiar ideas y conocimientos, la actualización profesional, el trabajo en equipo y el conocimiento de las líneas de investigación de las compañeras y sus aportaciones.

Entre los beneficios que los estudiantes pueden haber disfrutado, las co-docentes han señalado también elementos tanto académicos (e.g. formativos, perspectivas, contenidos, vivencias, conexión entre teoría y práctica) como elementos más personales e interpersonales tales como la confianza y entusiasmo transmitida por todos los implicados y la posibilidad de crear vínculos que supongan apoyos en su formación, como expresaba una profesora en la siguiente cita:

"La interacción entre iguales como instrumento de intercambio de información y conocimiento, así como de creación de vínculo de apoyo educativo".

Por otra parte, los estudiantes que ejercieron el rol de mentores han visto favorecidas sus habilidades sociales, su sentimiento de competencia autopercibida en relación a la temática que estaban explicando y su satisfacción al sentirse escuchados y valorados. Algunas manifestaciones que lo evidencian son los siguientes fragmentos:

"Me ha ayudado a explicarme mejor en grupo hacia gente desconocida"; "No solo me he sentido competente sino que también me ha servido para darme cuenta de que realmente creo y confio en lo que estoy explicando".

Por su parte, los estudiantes mentorizados destacan el conocimiento profundo adquirido sobre la temática abordada y un mayor conocimiento del plan de estudios y la organización de las materias que tendrán en futuros cursos. Así lo dicen:

"Tener una idea de a lo que nos enfrentaremos el curso que viene, qué aspectos tenemos que tener en cuenta".

\subsection{Intención de compromiso en actividades de co-docencia}

Las reacciones de las co-docentes ante la cuestión de si volverían a desarrollar una experiencia de co-docencia han sido afirmativas, aunque han diferido en la intensidad y rotundidad con la que lo han expresado. Desde una clara afirmación apoyada en la consideración de sus resultados positivos por haber favorecido el diálogo entre los estudiantes y el profesorado; pasando por una afirmación con ligeras matizaciones ("esta vez ha sido una experiencia breve, por supuesto me entusiasma la idea de poder desarrollarla en todas sus dimensiones"), a la expresión de los motivos por los que no podría hacerlo ("mis horarios lectivos no me permiten encontrar momentos para compartir con otros colegas") que por otra parte tampoco denota una falta de interés.

\subsection{Propuestas para la co-docencia en la Educación Superior}

De las dos últimas cuestiones planteadas a las profesoras, relativas a qué cambios propondrías y cuándo recomendarías este tipo de experiencias, extraemos información de interés para la puesta en marcha de la co-docencia en la Educación Superior.

(cc) EY-NC-ND 2017, Universitat Politècnica de València 
En cuanto a los cambios que propondrías las co-docentes señalan la necesidad de disponer de más tiempo para realizar co-docencia (para su planificación y desarrollo de forma efectiva), así como de apoyo por parte del decanato a través de "proyectos compartidos".

Por otra parte, hemos detectado un acuerdo en la propuesta de co-docencia en el Practicum y TFG, además de en asignaturas cuyos contenidos suponen procesos en espiral a lo largo de los cursos, o para desarrollar competencias básicas como "escribir para aprender y aprender a escribir". No obstante, una de las docentes considera que es muy complicada la co-docencia en la Educación Superior dadas la características actuales del Plan de estudios.

\section{Conclusiones}

En este trabajo se ha presentado el diseño y la valoración de una experiencia de innovación docente que combina el enfoque de co-docencia en la modalidad de equipo docente con la co-docencia en estaciones planteada esta última a través de la mentoría entre estudiantes.

La valoración de la experiencia realizada por parte de las co-docentes participantes refleja los beneficios apuntados ya por autores como Chanmugam y Gerlach (2013), Crow y Smith (2005) y Rodríguez (2014), presentando a la práctica de co-docencia como un medio posibilitador de formación, intercambio de reflexiones, de conocimiento de otras perspectivas y mejora de la competencia interpersonal y personal. En este sentido, las docentes participantes señalaron un incremento en su nivel de seguridad y autoconfianza. Además en nuestro estudio se identifican beneficios para los estudiantes percibidos por el profesorado tanto a nivel académico como personal vinculados con un mayor conocimiento de las conexiones existentes entre las asignaturas así como la creación de vínculos de apoyo educativo entre el alumnado participante. Se triangula esta valoración con los resultados del estudio de Castro, Izquierdo-Magaldi y García-Ruiz (2017) dónde los mismos estudiantes vienen a confirmar los beneficios percibidos por estas docentes.

No obstante, también se han identificado limitaciones propias de la práctica de co-docencia señaladas también por Castro y Figueroa (2006); Ginther, Phillips, y Grinseki (2007) y Rodríguez (2014) tales como la dificultad de la gestión de tiempos y espacios, el trabajo individualista. En nuestro estudio se ha identifico también la dificultad para llegar a un diseño que incluya dentro del proceso de enseñanza-aprendizaje una evaluación que implique la participación de todos los participantes. A nivel de su desarrollo dentro del contexto de la Educación Superior además de los elementos limitadores anteriormente apuntados se señalan, la necesidad de compromiso para mantener el esfuerzo y dedicación que conlleva una experiencia continuada de co-docencia.

Estas limitaciones no suponen un obstáculo para propuestas destinadas a la generalización de estas actividades en la Educación Superior si bien, en todas ellas se manifiesta la necesidad de contar con el respaldo y apoyo de la Administración para que facilite los tiempos, los espacios y la flexibilidad del currículo. 


\section{Referencias}

ÁlVAREZ-ARREGUI, E., RODRIGUEZ, A., MADRIGAL, R., GROSSI, B.A., Y ARREGUIT, X. (2017). "Ecosistemas de formación y competencia mediática: Valoración internacional sobre su implementación en la educación superior". Comunicar, Vol. 51, preprint.

BEAMISH, W., BRYER, F. y DAVIES, M. (2006). "Teacher reflections on co-teaching a unit of work". International Journal of Whole Schooling, Vol. 2 (2), pp. 3 - 18

BEKERMAN, D. y DANKNER, L. (2010). "La pareja pedagógica en el ámbito universitario, un aporte a la didáctica colaborativa". Formación Universitaria, Vol. 3 (6), pp. $3-8$.

CASTRO, R. y FIGUEROA, B. (2006). “Trabajo colaborativo en escuelas especiales de lenguaje, un enfoque educativo curricular". Paideia, Vol.41, pp. 117-127.

CASTRO, A., IZQUIERDO-MAGALDI, B. y GARCÍA-RUIZ, R. (2017). "Innovación docente en la universidad mediante co-docencia y mentoría". Comunicación presentada en el XIV Congreso Internacional de Educación Inclusiva, Oviedo.

CHANMUGAM, A. y GERLACH, B. (2013). "A Co-Teaching Model for Developing Future. Educators". Teaching Effectiveness International Journal of Teaching and Learning in Higher Education, Vol. 25 (1), pp. 110-117. $<$ http://www.isetl.org/ijtlhe/pdf/IJTLHE1412.pdf $>$ [Consulta: 15 de octubre de 2016]

CONDERMAN, G. y HEDIN, L. (2012). "Purposeful assessment practices for coteachers". Teaching Exceptional Children, Vol. $44 \quad$ (4), pp. 19 - 27. $<$ http://studentimpact.wmwikis.net/file/view/Assessment $\% 20$ for $\% 20 \mathrm{Co}-$ teachers.pdf/433004592/Assessment\%20for\%20Co-teachers.pdf $>$ [Consulta: 5 de octubre de 2016]

COOK, L. y FRIEND, M. (1995). "Co-teaching: guidelines for creating effective practices". Focus on Exceptional Children, Vol. 28(3), pp. 1-25. $<$ http://plaza.ufl.edu/mrichner/Readings/Cook\%20\&\%20Friend\%20(1995).pdf> [Consulta: 20 de enero de 2017]

CROW, J., y SMITH, L. (2003). "Using co-teaching as a means of facilitating interprofessional collaboration in health and social care". Journal of Interprofessional Care, Vol. 17(1), pp. 45-55.

FLECHA, R. et al. (2014). "Actuaciones de Éxito en la Universidad. Hacia la excelencia tomando las mejores universidades como modelo". Multidisciplinary Journal of Educational Research, Vol.4(2), pp. 131-150.

FRIEND, M. y COOK., L. (1996). Interactions: collaboration skills for school professionals. White Plains: Longman Publisher USA.

GINTHER, S. D., PHILLIPS, A., y GRINESKI, S. (2007). "Team-teaching the HBSE curriculum: Considerations and advice". Journal of Teaching in Social Work, Vol. 27(1-2), pp.199-211.

HUGHES, C. y MURAWSKI, W. (2001). "Lessons from another field: applying coteaching strategies to gifted education". Gifted Child Quarterly, Vol. 45 (3), pp. 195 203. 
PICHEIRA, J y OTONDO, M. (2016). "Habilidades de co-docencia en alumnos de Pedagogía en Educación Diferencial de la Universidad Católica de la Santísima Concepción". Revista de Estudios y Experiencias en Educación, Vol. 15 (29), pp. 95 - 108.

RODRÍGUEZ, F. (2014). "La co-enseñanza una estrategia para el mejoramiento educativo y la inclusion". Revista Latinoamericana de inclusión educativa, Vol. 8 (2), pp. 219-233.

SUÁREZ-DÍAZ, G. (2016). "Co-enseñanza: concepciones y prácticas en profesores de una Facultad de Educación en Perú”. Revista Electrónica de Investigación Educativa, Vol.18(1), pp.166-182. <http://redie.uabc.mx/redie/article/view/786> [Consulta: 10 de marzo de 2017]

TOBIN, K. y ROTH, W. (2005). "Implementing coteaching and cogenerative dialoguing in urban science education". School Science and Mathematics, Vol. 105 (6), pp. 313 - 321.

VALVERDE, A., RUIZ DE MIGUEL, C., GARCÍA JÍMENEZ, E. y ROMERO, S. (2004). "Innovación en la orientación universitaria: la mentoría como respuesta". Contextos educativos, Vol. 6-7, pp. 87-112.

VILLA, R. A., THOUSAND, J. S. y NEVIN, A. I. (Eds.). (2008). A guide to co-teaching: practical tips for facilitating student learning. Thousands Oaks, CA: Corwin Press. 